\title{
Case Report: A Case of Severe Cryptococcal Immune Reconstitution Inflammatory Syndrome Presenting with Brain and Intradural Abscesses in an HIV Patient
}

\author{
Thomas Kalinoski, ${ }^{1}$ Jason Malenfant, ${ }^{2}$ Catherine Yim $^{3}$ and Arthur Jeng ${ }^{2 \star}$ \\ ${ }^{1}$ Department of Internal Medicine, Olive View - UCLA Medical Center, Sylmar, California; ${ }^{2}$ Division of Infectious Diseases, Olive View - UCLA \\ Medical Center, Sylmar, California; ${ }^{3}$ Department of Radiology, Olive View - UCLA Medical Center, Sylmar, California
}

\begin{abstract}
Clinical worsening or new manifestation of cryptococcal disease following initiation of anti-retroviral therapy (ART) in an HIV patient is a hallmark of cryptococcal immune reconstitution inflammatory syndrome (C-IRIS). However, it can be difficult to distinguish IRIS from worsening or new infection. Here, we present a case of severe C-IRIS involving multiple cerebellar, spinal, and intradural abscesses and spinal arachnoiditis 7 months after ART initiation in an AIDS patient with uncertain prior ART compliance. He had multiple prior episodes of cryptococcal meningitis with complications necessitating ventriculoperitoneal shunt placement and was on suppressive fluconazole when he developed worsening brain manifestations. He received empiric anti-cryptococcal re-induction without improvement. All cerebrospinal fluid cultures remained sterile, with negative Cryptococcus PCR testing, and his condition continued to worsen prior to corticosteroid initiation. Ultimately, C-IRIS was diagnosed by brain biopsy. This case demonstrates an extreme in severity of C-IRIS and in the timeline of presentation after ART initiation.
\end{abstract}

\section{INTRODUCTION}

Immune reconstitution inflammatory syndrome due to Cryptococcus cryptococcal immune reconstitution inflammatory syndrome (C-IRIS) occurs in $19.5 \%$ of HIV patients with priordocumented AIDS-defining conditions starting anti-retroviral therapy $(\mathrm{ART})^{1}$ and remains a significant cause of morbidity and mortality in this population. Cryptococcal immune reconstitution inflammatory syndrome presents after beginning ART as either worsening of previously diagnosed cryptococcal meningitis or as an "unmasking" of previously unknown cryptococcal disease. ${ }^{2}$ Cryptococcal immune reconstitution inflammatory syndrome normally presents as meningitis, but other central nervous system (CNS) manifestations include intracranial cryptococcomas, cysts, abscesses, hydrocephalus, and raised intracranial pressure, cranial nerve lesions, dysarthria, and paresis. ${ }^{2-14}$ Immune reconstitution inflammatory syndrome may present similarly to disease relapse, which can make diagnosis challenging. ${ }^{15} \mathrm{We}$ describe a case of an AIDS patient with recurrent cryptococcal meningitis who developed CNS C-IRIS after ART initiation, the severity and extent of which has not been previously reported.

\section{CASE REPORT}

A 48-year-old man with HIV/AIDS and recurrent Cryptococcus neoformans meningitis with obstructive hydrocephalus requiring ventriculoperitoneal shunt (VPS) on maintenance fluconazole and ART presented with 4 months of progressive confusion, photophobia, and weakness. The patient had five prior episodes of cryptococcal meningitis, most recently 8 months prior (CD $4<20$ cells $/ \mu \mathrm{L}$ at that time) in the setting of poor medication compliance, and multiple social barriers to routine follow-up care. He had been treated with amphotericin B and flucytosine for 2 weeks, and then continued on fluconazole for consolidative therapy. Anti-retroviral therapy was started 24 days after antifungal initiation.

*Address correspondence to Arthur Jeng, Division of Infectious Diseases, Olive View - UCLA Medical Center, 14445 Olive View Dr., Sylmar, CA 91342. E-mail: ajeng@dhs.lacounty.gov
At the time of presentation, 7 months after ART initiation, he was started on amphotericin B plus flucytosine. His ART was continued, as CD4 had improved to 35 cells $/ \mu \mathrm{L}$ and HIV viral load was undetectable. Lumbar cerebrospinal fluid (CSF) studies revealed white blood cells count (WBC) 72 cells/cumm, total protein $620 \mathrm{mg} / \mathrm{dL}$, glucose $35 \mathrm{mg} / \mathrm{dL}$, and CrAg titer > 1: 2,560. Fungal culture and Cryptococcus PCR were negative. Magnetic resonance imaging (MRI) of the brain showed leptomeningeal enhancement and micro-abscesses (Figure 1).

After 2 weeks of induction therapy, repeat CSF studies showed opening pressure $32 \mathrm{cmH}_{2} \mathrm{O}$, WBCs 127 cumm, total protein $620 \mathrm{mg} / \mathrm{dL}$, and glucose $39 \mathrm{mg} / \mathrm{dL}$; fungal culture remained negative. A second MRI showed new ring-enhancing abscesses in the cerebellum, worsening leptomeningeal enhancement with occlusion of the fourth ventricle, and new vasogenic edema in the temporal lobe (Figure 2).

The diagnoses considered included refractory Cryptococcus, C-IRIS, tuberculous (TB) meningitis, or bacterial VPS infection. He was started on empiric TB therapy (rifampin/isoniazid/pyrazinamide/ ethambutol or RIPE) plus dexamethasone while CSF AFB cultures were pending, and also placed on meropenem and vancomycin. $\mathrm{He}$ continued to decline neurologically, prompting neurosurgery consultation for brain biopsy. All bacterial, fungal, and acid fast bacilli (AFB) cultures of the left frontal bone, dura, arachnoid, and brain were negative. Pathology showed numerous yeast consistent with Cryptococcus (Figure 3). Given the presence of yeast and negative cultures, C-IRIS was considered the most likely diagnosis. The lack of response to antifungal, anti-TB, and antibiotic therapy supported this. Prednisone $40 \mathrm{mg}$ daily was started, and fluconazole and RIPE were continued.

At his follow-up visit, he was neurologically stable, and prednisone was decreased to $35 \mathrm{mg}$ daily. Three weeks later, fevers, headaches, weakness, and bowel/bladder incontinence recurred. CD4 count was 46 cells/ $\mu \mathrm{L}$ (6\%). Cerebrospinal fluid showed WBCs 1900 cells/cumm, total protein $620 \mathrm{mg} / \mathrm{dL}$, and glucose 10 $\mathrm{mg} / \mathrm{dL}$; all cultures remained negative (Table 1). MRI of thoracic and lumbar spine showed dural thickening and enhancement throughout the spine (Figure 4). This included areas of dorsal phlegmon in the thoracic region and a complex abscess surrounding the thecal sac, compressing the S1 nerve root. CTguided aspiration of this abscess revealed 1+ yeast and 1+PMNs 

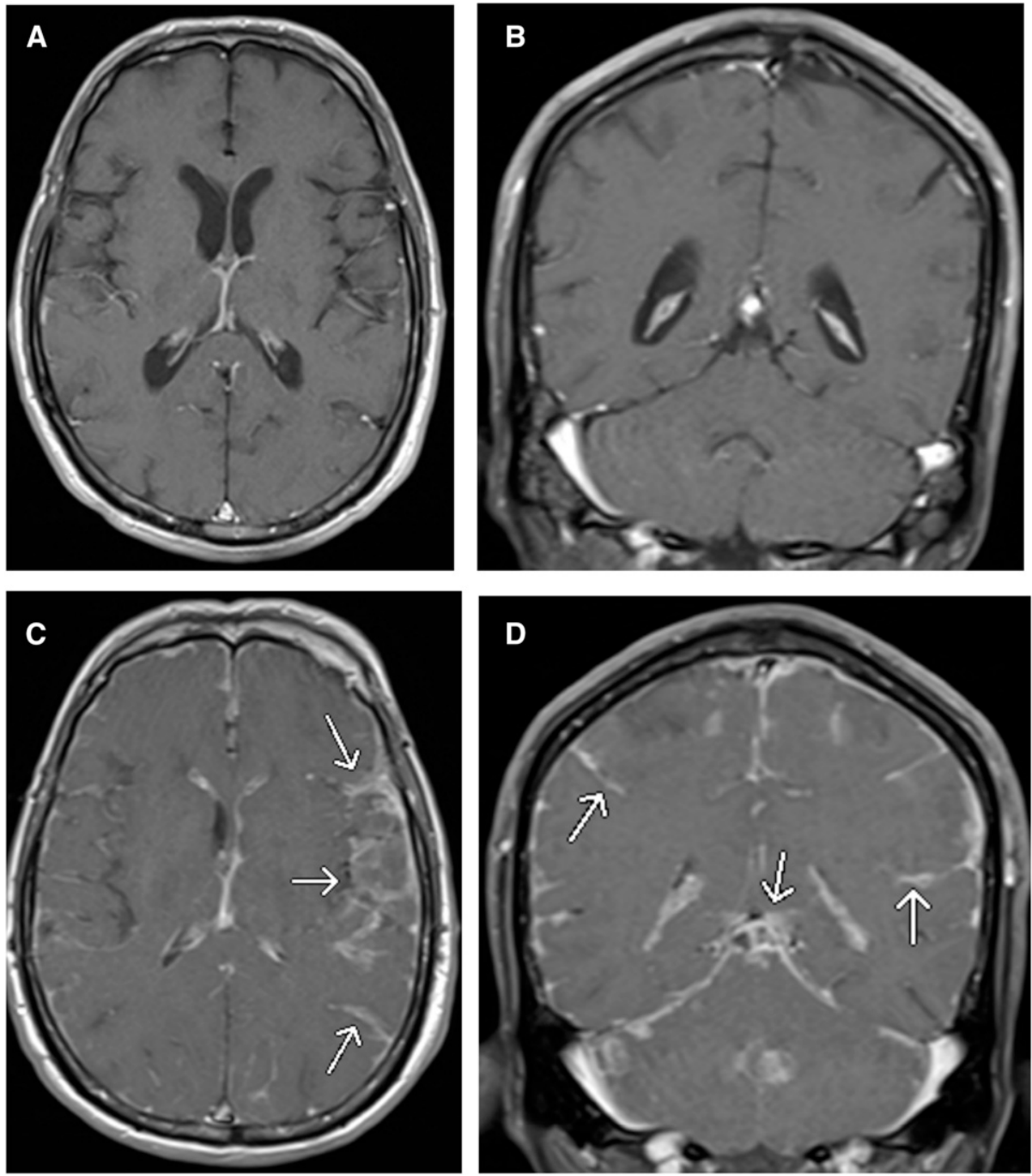

FIGURE 1. Initial axial (A) and coronal (B) contrast-enhanced T1-weighted MRI images show no abnormal enhancement. Subsequent axial (C) and coronal (D) contrast-enhanced T1-weighted MRI images show new diffuse leptomeningeal enhancement (arrows).

on Gram stain but no growth in cultures. With multiple negative cultures, yet still a moderate burden of yeast on staining, the suspicion for C-IRIS further increased, and we concluded that his clinical deterioration likely correlated with tapering his corticosteroid dosing. Thus, his prednisone was increased to $60 \mathrm{mg}$ daily. Because of remaining uncertainty regarding occult, active cryptococcal disease, the patient was restarted on flucytosine for 2 weeks, while continuing on maintenance fluconazole (400 mg daily). His RIPE therapy was discontinued after the AFB cultures of CSF demonstrated no growth at 6 weeks.

With the increased corticosteroid dosing, he exhibited sustained clinical improvement over time. He was maintained on prednisone $60 \mathrm{mg}$ daily for 3 weeks and $55 \mathrm{mg}$ daily for 6 weeks, which was reduced to $50 \mathrm{mg}$ daily at his most recent follow-up. At this appointment, 2 months following his hospital course, he continued to have significant improvement in his neurological status.

\section{DISCUSSION}

Cryptococcal meningitis remains among the most common causes of AIDS-related mortality, estimated to be $15-20 \%$. $^{16}$
Cryptococcal immune reconstitution inflammatory syndrome is a common complication of cryptococcal meningitis following ART initiation, and the two disorders can be difficult to distinguish. This case was particularly challenging because of extensive brain and spinal disease, which is more often seen with severe TB or Coccidioides CNS infections. Chronic hydrocephalus and a VPS further complicated the possible diagnosis.

Cerebrospinal fluid inflammation is nonspecific in this setting and is consistent with Cryptococcus meningitis, C-IRIS, cytomegalovirus polyradiculopathy, or another infection, such as tuberculosis, bacterial, or rarer forms of meningitis. The patient's CrAg titer remained high in all CSF samples, but these levels can remain elevated despite resolving or resolved infection, and serial monitoring has a limited diagnostic value. $^{17}$ The multiple negative CSF cultures and Cryptococcus PCR assays support an inflammatory disease process rather than active infection. The brain biopsy strongly supported diagnosis of C-IRIS, with pathology showing Cryptococcus yeast with negative cultures, leading to the initiation of corticosteroids. 

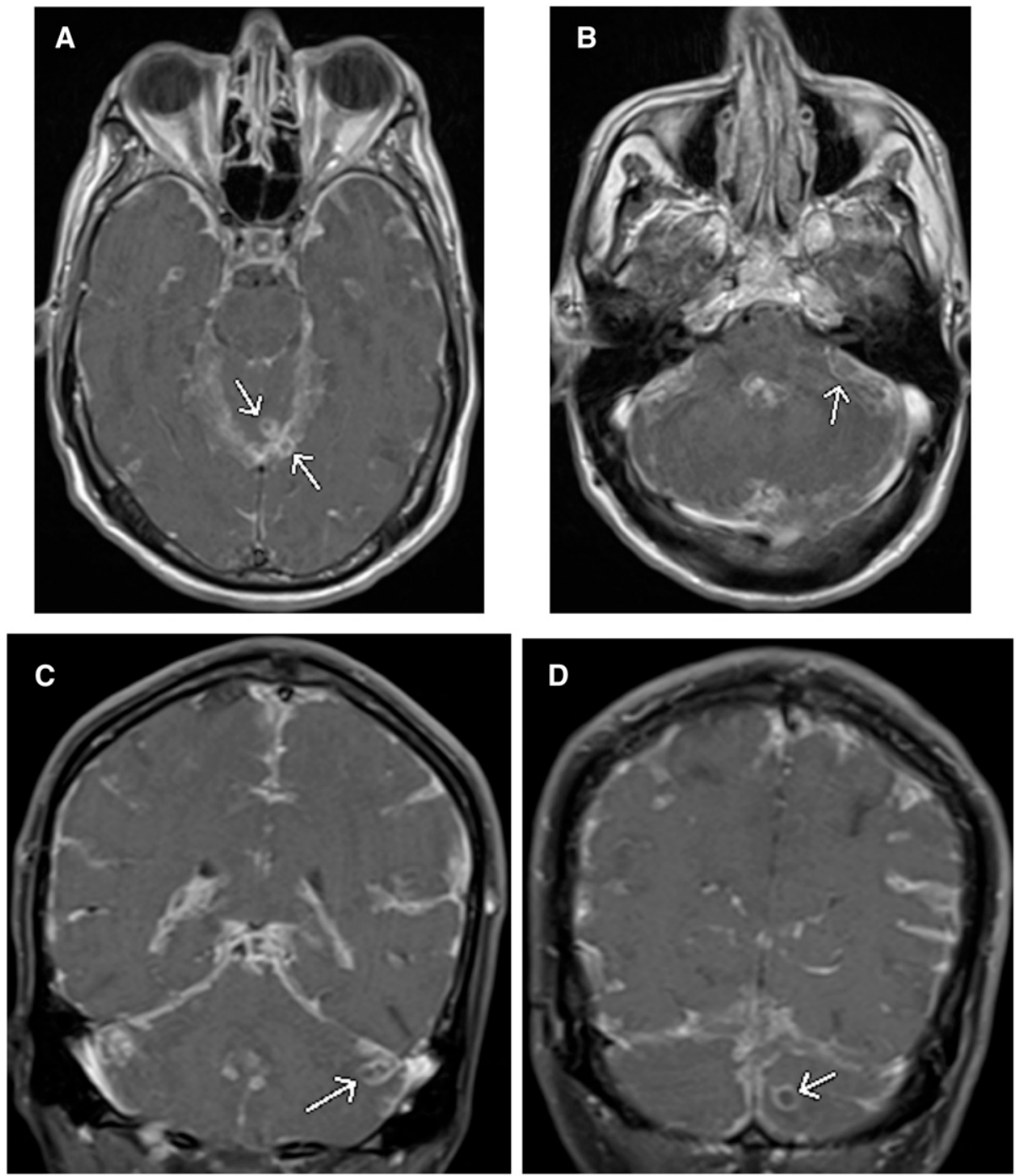

Figure 2. Follow-up axial (A and B) and coronal (C and $\mathbf{D})$ contrast-enhanced T1-weighted MRI images show worsened leptomeningeal enhancement and new ring-enhancing parenchymal abscesses abutting the tentorium and within the left cerebellar hemisphere (arrows).

A limited number of cases of isolated infratentorial or spinal cryptococcal infection have been reported. The cerebellar cases were in immunocompetent patients. ${ }^{18,19}$ Spinal cases range from epidural involvement to intramedullary abscess. ${ }^{20-32}$ To our knowledge, only one case of spinalcerebral infection due to $C$. neoformans, which was in an HIVnegative, immunocompetent patient, has been reported. ${ }^{33}$ Ours is the first known case of C-IRIS with these widespread and specific manifestations. Prior cases of C-IRIS involving the spine may have been undiagnosed because it was asymptomatic. Indeed, even with extensive spinal involvement, our patient did not have significant back pain. Thus, we advise a low threshold for spine imaging when severe C-IRIS is suspected, especially if the CSF profile continues to be markedly abnormal.

Although the current standard is to start patients on ART as early as possible, even with ongoing opportunistic infection, delay is advised with certain infections. Studies on tuberculous meningitis have shown significantly higher severe adverse events with early ART ${ }^{34}$ In cryptococcal meningitis, one study showed that early ART (within 72 hours of cryptococcal meningitis diagnosis) in patients with AIDS resulted in significantly higher mortality than delayed (after 10 weeks of fluconazole) ART (88\% versus $54 \%) .{ }^{35}$ A subsequent similar study found higher mortality in early (1-2 weeks) than in delayed ( 5 weeks after diagnosis) ART cohorts $(45 \%$ versus $30 \%)^{36}$

Current U.S. guidelines recommend ART be initiated 210 weeks after the start of antifungal therapy in HIV patients with cryptococcal meningitis. ${ }^{37,38}$ Given that studies have looked at only two intervals ( 5 and 10 weeks) for "delayed" therapy, the optimal time for ART initiation may be even longer, especially in cases of severe cryptococcal meningitis. Our patient was initiated on ART 24 days after starting antifungal therapy. We suspect he used ART only sporadically in the prior 7 months given his high levels of HIV viremia and low CD4 count. This case illustrates that even with successful treatment of cryptococcal meningitis and a delay in starting ART, patients may have life-threatening C-IRIS.

Patients with advanced AIDS remain at high risk for C-IRIS after the initiation of ART. It can be difficult to predict which of these patients may develop severe C-IRIS, even in instances 

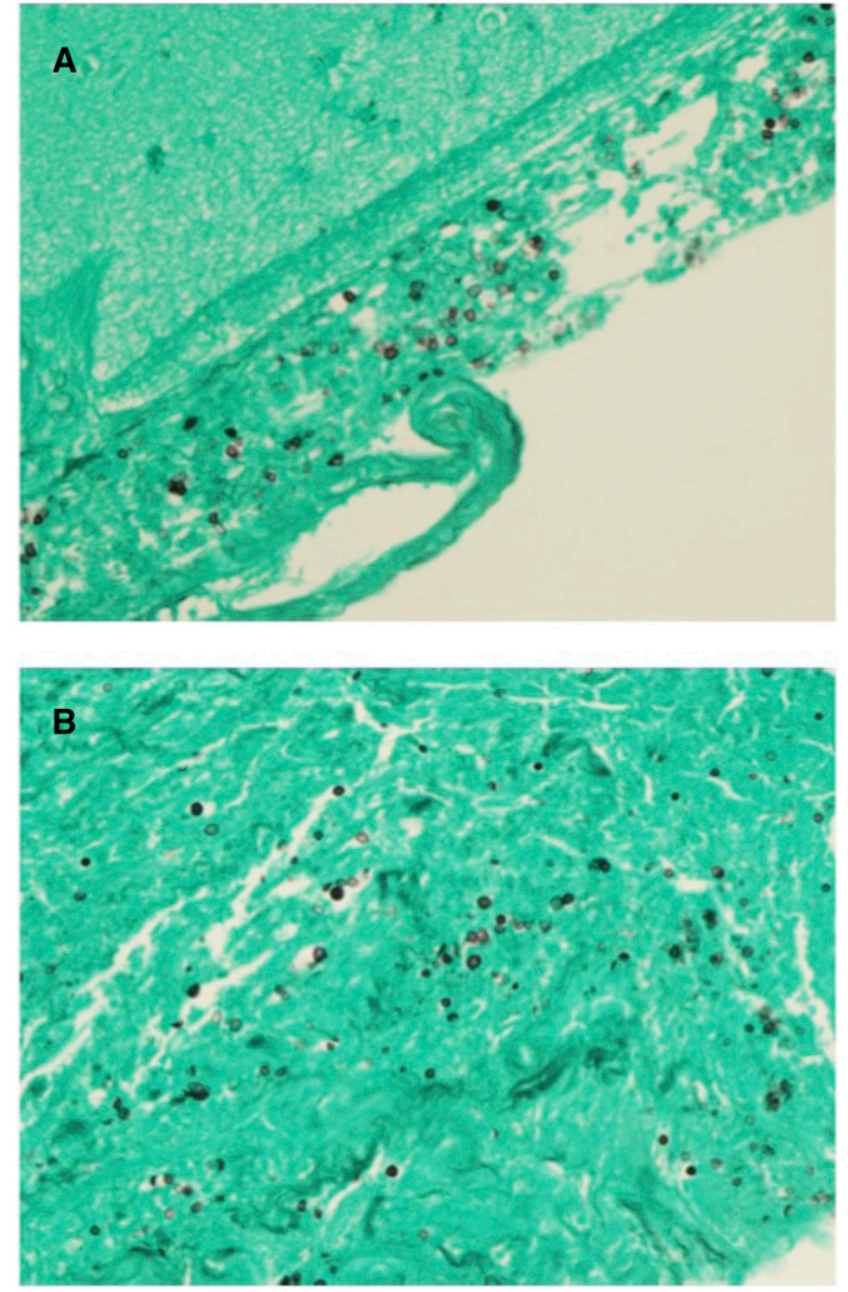

FIGURE 3. Grocott's methenamine silver (GMS)-stained pathology specimens of the brain (A) and arachnoid (B) tissues revealing numerous yeast forms. This figure appears in color at www.ajtmh.org.

of delaying ART initiation appropriately. Our patient had known risk factors associated with poorer outcomes in cryptococcal meningitis, such as high pre-ART CrAg and
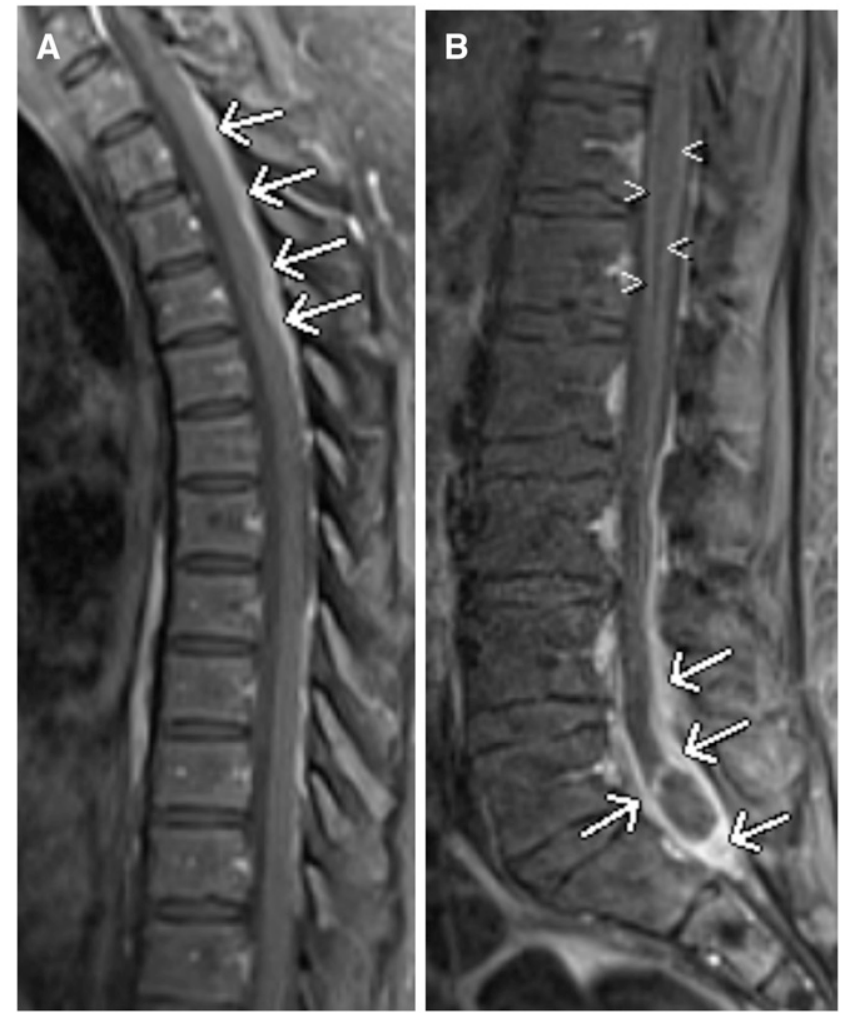

FIGURE 4. Sagittal contrast-enhanced T1-weighted MRI image through the thoracic spine (A) shows dorsal dural thickening and enhancement (arrows). Sagittal contrast-enhanced T1-weighted MRI image through the lumbar spine (B) shows leptomeningeal enhancement around the conus (arrowheads) and dural thickening and enhancement around the distal thecal sac (arrows).

lower levels of CSF inflammation. ${ }^{39-42}$ However, this pattern is not uncommon in patients with cryptococcal meningitis in advanced AIDS. His absolute CD4 count did not appreciably increase after ART initiation (Table 1), which may give a false impression that IRIS was not occurring. However, his CD4\% did markedly increase from $1 \%$ to $6-7 \%$, making the percentage, perhaps, a more sensitive marker for IRIS, as shown in prior studies. ${ }^{43,44}$

TABLE 1

Serum and CSF parameters at indicated time points before and after ART initiation

\begin{tabular}{|c|c|c|c|c|c|c|c|c|c|c|}
\hline \multicolumn{6}{|c|}{ Pre-ART initiation* ${ }^{*}$} & \multicolumn{5}{|c|}{ Post-ART initiation } \\
\hline Date & $\begin{array}{l}\text { October } \\
15,2017\end{array}$ & $\begin{array}{l}\text { October } \\
21,2017\end{array}$ & $\begin{array}{l}\text { October } \\
24,2017\end{array}$ & $\begin{array}{l}\text { October } \\
26,2017\end{array}$ & $\begin{array}{l}\text { October } \\
28,2017\end{array}$ & $\begin{array}{c}\text { February } \\
9,2018\end{array}$ & $\begin{array}{l}\text { June 11, } \\
2018\end{array}$ & $\begin{array}{l}\text { June } 22 \\
2018\end{array}$ & $\begin{array}{l}\text { June } 27 \\
2018\end{array}$ & $\begin{array}{c}\text { August 29, } \\
2018\end{array}$ \\
\hline CD4 (cells/ $\mu \mathrm{L}$ ) & & & $<20$ & & & 40 & & 35 & & 46 \\
\hline CD4 (\%) & & & 1 & & & 6 & & 7 & & 6 \\
\hline LP OP $\left(\mathrm{cm} \mathrm{H}_{2} \mathrm{O}\right)$ & 24 & 50 & 36 & 47 & 39 & - & - & 29 & 32 & - \\
\hline $\begin{array}{l}\text { CSF WBC (per } \\
\text { cumm) }\end{array}$ & 2 & 5 & 3 & 0 & 12 & 96 & 72 & 187 & 127 & 1900 \\
\hline CSF neut (\%) & 3 & 12 & 5 & - & 0 & 21 & 25 & 65 & 52 & 74 \\
\hline CSF lymph (\%) & 61 & 65 & 39 & - & 43 & 70 & 67 & 25 & 34 & 16 \\
\hline $\begin{array}{l}\text { CSF total protein } \\
(\mathrm{mg} / \mathrm{dL})\end{array}$ & 36 & 34 & 27 & 33 & 28 & 620 & 620 & 620 & 620 & 620 \\
\hline CSF glucose (mg/dL) & 13 & 17 & 20 & 19 & 24 & 10 & 35 & 41 & 39 & 10 \\
\hline CSF CrAg titer & $1: 2,560$ & $1: 2,560$ & $1: 2,560$ & $1: 2,560$ & $1: 2,560$ & $1: 2,560$ & $1: 2,560$ & $1: 2,560$ & - & $1: 2,560$ \\
\hline C. neo. PCR & Positive & - & - & - & - & Negative & Negative & - & - & Negative \\
\hline CSF Gram stain & $\begin{array}{c}\text { Moderate } C . \\
\text { neo. }\end{array}$ & $\begin{array}{c}\text { Moderate } C . \\
\text { neo. }\end{array}$ & $\begin{array}{c}\text { Moderate } C . \\
\text { neo. }\end{array}$ & $\begin{array}{c}\text { Moderate } C . \\
\text { neo. }\end{array}$ & $\begin{array}{c}\text { Rare } C . \\
\text { neo. }\end{array}$ & $\begin{array}{c}\text { Moderate } C . \\
\text { neo. }\end{array}$ & Negative & Negative & Negative & Negative \\
\hline CSF fungal culture & C. neo. & Negative & C. neo. & - & C. neo & Negative & Negative & Negative & Negative & Negative \\
\hline
\end{tabular}

$\mathrm{ART}=$ anti-retroviral therapy; $\mathrm{C}$. neo $=$ Cryptococcus neoformans; $\mathrm{CSF}=$ cerebrospinal fluid; $\mathrm{OP}=$ opening pressure

${ }^{*}$ Anti-retroviral therapy initiation November 9, 2017. 
This case demonstrates that complications of C-IRIS can include cerebellar abscess and spinal involvement, the latter of which may occur without significant localizing symptoms. Distinguishing such manifestations from worsening cryptococcal infection or coinfection can be challenging, and biopsies of the affected areas may be warranted for definitive determination. The extremes in severity and time course of this case reinforces previous research on the benefit of delayed ART initiation for cryptococcal IRIS up to 10 weeks from the time of antifungal initiation, especially for patients with certain predictive factors of IRIS as mentioned earlier. An individual risk/ benefit profile should be considered by the treating clinician, further adding to the complexity in caring for these often-challenging and gravely ill patients.

Received January 12, 2020. Accepted for publication March 23, 2020. Published online April 27, 2020.

Acknowledgment: We thank Wenchang Guo, the pathology contributor.

Authors' addresses: Thomas Kalinoski, Department of Internal Medicine, Olive View - UCLA Medical Center, Sylmar, CA, E-mail: tkalinoski@dhs.lacounty.gov. Jason Malenfant and Arthur Jeng, Division of Infectious Diseases, Olive View - UCLA Medical Center, Sylmar, CA, E-mails: jason.malenfant@gmail.com and ajeng@ dhs.lacounty.gov. Catherine Yim, Department of Radiology, Olive View - UCLA Medical Center, Sylmar, CA, E-mail: cyim@ dhs.lacounty.gov.

\section{REFERENCES}

1. Müller $M$, Wandel $S$, Colebunders $R$, Colebunders $R$, Attia $S$, Furrer H, Egger M, 2010. Immune reconstitution inflammatory syndrome in patients starting antiretroviral therapy for HIV infection: a systematic review and meta - analysis. Lancet Infect Dis 10: 251-261.

2. Haddow LJ et al.; International Network for the Study of HIV-associated IRIS (INSHI), 2010. Cryptococcal immune reconstitution inflammatory syndrome in HIV-1-infected individuals: proposed clinical case definitions. Lancet Infect Dis 10: 791-802.

3. Rambeloarisoa J, Batisse D, Thiebaut J, Mikol J, Mrejen S, Karmochkine M, Kazatchkine MD, Weiss L, Piketty C, 2002. Intramedullary abscess resulting from disseminated cryptococcosis despite immune restoration in a patient with AIDS. $J$ Infect 44: 185-188.

4. Hu Z, Wei H, Meng F, Xu C, Cheng C, Yang Y, 2013. Recurrent cryptococcal immune reconstitution inflammatory syndrome in an HIV-infected patient after anti-retroviral therapy: a case report. Ann Clin Microbiol Antimicrob 12: 40.

5. Nunnari G, Gussio M, Pinzone MR, Martellotta F, Cosentino S, Cacopardo B, Celesia BM, 2013. Cryptococcal meningitis in an HIV-1-infected person: relapses or IRIS? Case report and review of the literature. Eur Rev Med Pharmacol Sci 17: 1555-1559.

6. Hashimoto H, Hatakeyama S, Yotsuyanagi H, 2015. Development of cryptococcal immune reconstitution inflammatory syndrome 41 months after the initiation of antiretroviral therapy in an AIDS patient. AIDS Res Ther 12: 33.

7. Pettersen KD, Pappas PG, Chin-Hong P, Baxi SM, 2015. A paradoxical decline: intracranial lesions in two HIV-positive patients recovering from cryptococcal meningitis. pii: bcr2015212108. Available at: https://doi.org/10.1136/bcr-2015-212108.

8. Ogbuagu O, Villanueva $M, 2014$. Extensive central nervous system cryptococcal disease presenting as immune reconstitution syndrome in a patient with advanced HIV: report of a case and review of management dilemmas and strategies. Infect Dis Rep 6: 5576.

9. Katchanov J, Blechschmidt C, Nielsen K, Branding G, Arasteh K, Tintelnot K, Meintjes G, Boulware DR, Stocker H, 2014. Cryptococcal meningoencephalitis relapse after an eight-year delay: an interplay of infection and immune reconstitution. Int $J$ STD AIDS 26: 912-914.

10. Malik A, Khan PA, Shujatullah F, Fatima N, Shameem M, Siddiqui $A, 2012$. Rapid development of IRIS in the form of cryptococcal meningitis after beginning ART. Med Mycol Case Rep 1: 56-58.

11. da Cunha Colombo ER, Mora DJ, Silva-Vergara ML, 2010. Immune reconstitution inflammatory syndrome (IRIS) associated with Cryptococcus neoformans infection in AIDS patients. Mycoses 54: 178-182.

12. Park JY, Kim MJ, 2014. Immune reconstitution inflammatory syndrome in acquired immune deficiency syndrome related to cryptococcal meningoencephalitis. J Invest Med High Impact Case Rep 2: 2324709614533951.

13. Kiggundu R, Rhein J, Meya DB, Boulware DR, Bahr NC, 2014. Unmasking cryptococcal meningitis immune reconstitution inflammatory syndrome in pregnancy induced by HIV antiretroviral therapy with postpartum paradoxical exacerbation. Med Mycol Case Rep 5: 16-19.

14. Musubire A, Meya B, Mayanja-Kizza H, Lukande R, Wiesner L, Bohjanen $\mathrm{P}$, Boulware RD, 2012. Challenges in diagnosis and management of Cryptococcal immune reconstitution inflammatory syndrome (IRIS) in resource limited settings. Afr Health Sci 12: 226-230.

15. Bahr N, Boulware DR, Marais S, Scriven J, Wilkinson RJ, Meintjes $G, 2013$. Central nervous system immune reconstitution inflammatory syndrome. Curr Infect Dis Rep 15: 583-593.

16. Park BJ, Wannemuehler KA, Marston BJ, Govender N, Pappas PG, Chiller TM, 2009. Estimation of the current global burden of cryptococcal meningitis among persons living with HIV/AIDS. AIDS 23: 525-530.

17. Powderly WG, Cloud GA, Dismukes WE, Saag MS, 2014. Measurement of cryptococcal antigen in serum and cerebrospinal fluid: value in the management of AIDS-associated cryptococcal meningitis. Clin Infect Dis 18: 789-792.

18. Gologorsky Y, Delamora P, Souweidane MM, Greenfield J, 2007. Cerebellar cryptococcoma in an immunocompetent child. J Neurosurg Pediatr 107: 314-317.

19. Kaya S, Köksal I, Tosun I, Sari A, Alioglu Z, 2012. Cryptococcal meningitis with accompanying recurrent cerebellitis in an immunocompetent patient. Med Mycol Case Rep 1: 127-129.

20. Litvinoff J, Nelson M, 1978. Extradural lumbar cryptococcosis. J Neurosurg 49: 921-923.

21. Gumbo T, Hakim JG, Mielke J, Siwji S, Just-Nubling G, Ismail A, 2001. Cryptococcus myelitis: atypical presentation of a common infection. Clin Infect Dis 32: 1235-1236.

22. Asanuma $Y$ et al., 2014. Extradural cryptococcoma at the sacral spine without bone involvement in an immunocompetent patient. J Orthop Sci 19: 1040-1045.

23. Houda B, Wafa A, Zoubida T, Mohamed A, Mohamed A, Hicham $H, 2011$. Vertebral cryptococcosis in an immunocompetent patient-a case report. Pan Afr Med J 8: 42.

24. Wang C, Jia N, Zhang L, Liu K, Liu H, Yu H, 2014. Imaging findings of cryptococcal infection of the thoracic spine. Int $J$ Infect Dis 29: 162-165.

25. Searls E, Sico JJ, Bulent Omay S, Bannykh S, Kuohung V, Baehring J, 2009. Unusual presentations of nervous system infection by Cryptococcus neoformans, Clin Neurol Neurosurg 111: 638-642.

26. Deus-Silva L, Costa AE, Bevilacqua JM, Assis DB, Ferraz CA, Oliveira A, Nucci A, 2004. Meningoradiculitis due to Cryptococcus neoformans in an immunocompetent patient. Arq Neuropsiquiatr 62: 147-149.

27. Aganovic L, Hoda RS, Rumboldt Z, 2004. Hyperintensity of spinal Cryptococcus infection on diffusion-weighted MR Images. AJR Am J Roentgenol 183: 1176-1177.

28. Drouet A, Amah Y, Pavic M, Gerome P, Meyer X, Debourdeau, 2005. Méningoradiculomyéloencéphalite subaiguë liée à une infection par Cryptococcus neoformans. La Revue de Médecine Interne 26: 403-408.

29. Agrawal A, Agrawal A, Agrawal C, Rohtagi A, 2006. An unusual spinal arachnoiditis, Clin Neurol Neurosurg 108: 775-779.

30. Nfoussi H, Chelly I, Aamari L, Ben Salem T, Azouz H, Tiouiri Benaissia H, Kchir N, Haouet S, Zitouna M, 2010. Paraparesis and fever in a Tunisian woman: cryptococcal spondylitis with spinal involvement. Med Trop 70: 85-87. 
31. Gültaşli NZ, Ercan K, Orhun S, Albayrak S, 2007. MRI findings of intramedullary spinal cryptococcoma. Diagn Interv Radiol 13: 64-67.

32. Sloan DJ, Parris V, 2014. Cryptococcal meningitis: epidemiology and therapeutic options. Clin Epidemiol 6: 169-182.

33. Gupta R, Kushwaha S, Behera S, Jaiswal A, Thakur R, 2012. Vertebro-cerebral cryptococcosis mimicking tuberculosis: a diagnostic dilemma in countries with high burden of tuberculosis. Indian J Med Microbiol 30: 245-248.

34. Török ME et al., 2011. Timing of initiation of antiretroviral therapy in human immunodeficiency virus (HIV)-associated tuberculous meningitis. Clin Infect Dis 52: 1374-1383.

35. Makadzange AT, Ndhlovu CE, Takarinda K, Reid M, Kurangwa M, Gona P, Hakim JG, 2010. Early versus delayed initiation of antiretroviral therapy for concurrent HIV infection and cryptococcal meningitis in sub-saharan Africa. Clin Infect Dis 50: 1532-1538.

36. Boulware DR et al., 2014. Timing of antiretroviral therapy after diagnosis of cryptococcal meningitis. N Engl J Med 370:2487-2498.

37. Department of Health and Human Services, 2020. Panel on Antiretroviral Guidelines for Adults and Adolescents. Guidelines for the Use of Antiretroviral Agents in Adults and Adolescents with HIV. Available at: http://aidsinfo.nih.gov/contentfiles/ Ivguidelines/AdultandAdolescentGL.pdf. Accessed January 10, 2020.

38. Zolopa A, Andersen J, Powderly W, Sanchez A, Sanne I, Suckow C, Hogg E, Komarow L, 2009. Early antiretroviral therapy reduces AIDS progression/death in individuals with acute opportunistic infections: a multicenter randomized strategy trial. PLoS One 4: e5575.

39. Boulware DR, Meya DB, Bergemann TL, Wiesner DL, Rhein J, Musubire A, Lee SJ, Kambugu A, Janoff EN, Bohjanen PR, 2010. Clinical features and serum biomarkers in HIV immune reconstitution inflammatory syndrome after Cryptococcus meningitis: a prospective cohort study. PLoS Med 7: e1000384.

40. Sungkanuparph $S$ et al., 2009. Cryptococcal immune reconstitution syndrome after antiretroviral therapy in AIDS patients with cryptococcal meningitis: a prospective multicenter study. Clin Infect Dis 49: 931-934.

41. Boulware DR, Bonham SC, Meya DB, Wiesner DL, Park GS, Kambugu A, Janoff EN, Bohjanen PR, 2010. Paucity of initial cerebrospinal fluid inflammation in cryptococcal meningitis is associated with subsequent immune reconstitution inflammatory syndrome. J Infect Dis 202: 962-970.

42. Chang CC et al., 2013. Clinical and mycological predictors of cryptococcosis-associated immune reconstitution inflammatory syndrome. AIDS 27: 2089-2099.

43. Bonham S, Meya DB, Bohjanen PR, Boulware DR, 2008. Biomarkers of HIV immune reconstitution inflammatory syndrome. Biomark Med 2: 349-361.

44. Eshun-Wilson I, Okwen MP, Richardson M, Bicanic T, 2018. Early versus delayed antiretroviral treatment in HIV-positive people with cryptococcal meningitis. Cochrane Database Syst Rev 7. CD009012. 